\title{
A Design of Intelligent Management System for Warehouses
}

\author{
Xue Gou \\ Business school, Binzhou Polytechnic, Binzhou 256603, China \\ gouxue001@126.com
}

Keywords: Chip Microcomputer, Wireless Transmission, Liquid Crystal Display, Warehouse.

\begin{abstract}
The system is designed to assist administrators to realize intelligent management system for the warehouses of Large warehouse logistics enterprise. This system is divided into two parts: a console and warehouses. The console uses STM32 SCM as a main controller, and warehouse base on STC89C52 controller. The console and the warehouse connect with each other by NRF24L01 wireless transmission. The console uses the LCD screen to display the information, which facilitates the administrator to a plurality of remote management of warehouses.
\end{abstract}

\section{Introduction}

As an important component of enterprise operation, logistics is becoming more and more important. The warehouse is an important link in logistics, a large number of goods are stored in the warehouse, resulting in large and medium-sized enterprises or each of the third party logistics company will have a large number of warehouses, therefore, a plurality of warehouse manage workload is very large. At present, most enterprises mainly use artificial methods for warehouse management. And for a large number of warehouses for management, this will consume a lot of manpower, material resources, financial resources. When multiple warehouse will be opened at the same time within the scope of the case, the warehouse administrator can only run to open the door by the warehouse, resulting in reduced work efficiency. In view of the above situation, the system is designed to assist the administrator in the accurate and effective management of a large number of warehouses.

The system is suitable for warehouse logistics enterprises with large warehouses to manage. Because it uses a wireless remote control mode, the warehouse administrator can understand all the warehouses in the control room, and at the same time to control more than one warehouse, compared to the artificial way, which can reduce the cost of warehouse operation, also can make the work efficiency can be improved.

\section{Solution Design}

\subsection{Overall Design of the System}

This system is divided into two parts of the console and warehouse. The console uses STM32 as the controller, and the warehouse using STC89C52 microcontroller as the control core. Random password console STM32 will generate, through the wireless transmission module NRF24L01 to the corresponding warehouse, enter a temporary password in the micro controller STC89C52 of the warehouse will be entering the warehouse, at the same time display time and the number of entering the warehouse in the LCD screen on the console. The overall block diagram of the system is shown in figure 1. 


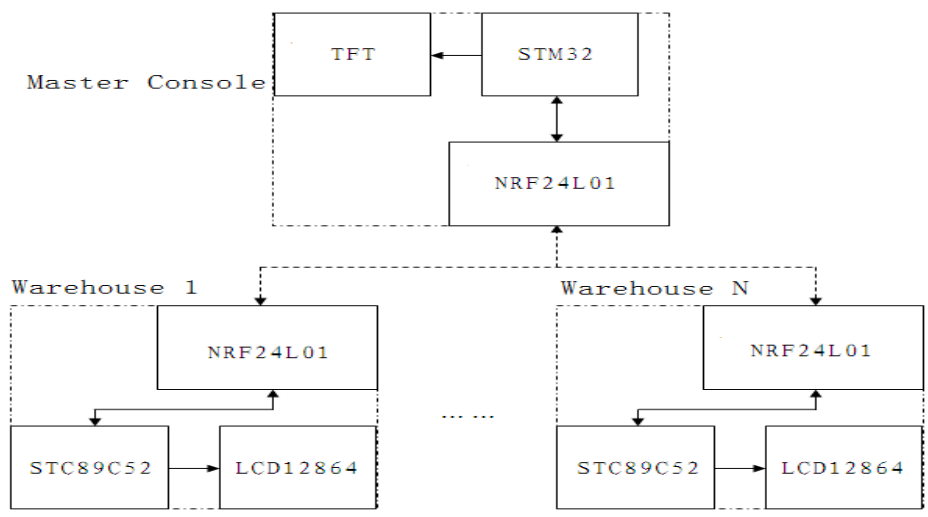

Fig. 1 Overall block diagram of the system

\subsection{Microcontroller Module Solution Selection}

First solution: ARM company developed STM32F106 medium capacity enhanced microcontroller, 32 bit ARM core, comes with 128K bytes flash memory, integrated USB, CAN, 7 timers, 2 ADC and 9 communication interfaces.

Second solution: STC89C52 microcontroller is a low-power, high-performance CMOS8 bit microcontroller, with $8 \mathrm{~K}$ in the system programmable Flash memory. With a smart 8 bit CPU and a system programmable Flash on a single chip, the STC89C52 provides a highly flexible and efficient solution for many embedded control applications.

Considering the selection of STM32F106 as a console controller, using STC89C52 as the MCU control warehouse.

\subsection{Data Transmission Module Solution Selection}

First solution: transmit data through RS232 transmission line. Although it is easy to monitor and easy to program, the data transmission distance is limited by the length of the connection, so the cost is too high when it is transmitted over long distance.

Second solution: using NRF24L01 wireless transceiver chip for data transmission, NRF24L01 wireless transceiver chip has the advantages of low power consumption, simple control, automatic processing prefix and CRC checksum, suitable for long distance transmission.

Because the system console with each warehouse distance, so the second solution is accepted.

\subsection{Display Module Solution Selection}

First solution: using TFT color display, the liquid crystal display large information capacity, and friendly interface, can display the image information and the touch screen.

Second solution: using LCD12864 graphics and Chinese dual purpose LCD display. Liquid crystal display, low power consumption, lightweight, shockproof. The interface circuit is simple, and can be directly connected with the microcontroller, showing rich information

Considering the console with TFT color, the warehouse using LCD12864 liquid crystal display.

\subsection{Final Solution}

After careful analysis and demonstration, the final solution of each module of the system is as follows:

(1) Micro controller module: console using STM32F106 microcontroller, the warehouse using STC89C52 microcontroller.

(2) Data transmission module: NRF24L01 wireless data transmission module.

(3) Display module: the console display module adopts TFT touch screen, the warehouse using LCD12864 liquid crystal display. 


\section{Design of System Hardware Circuit}

\subsection{Minimum System Console Controller Design}

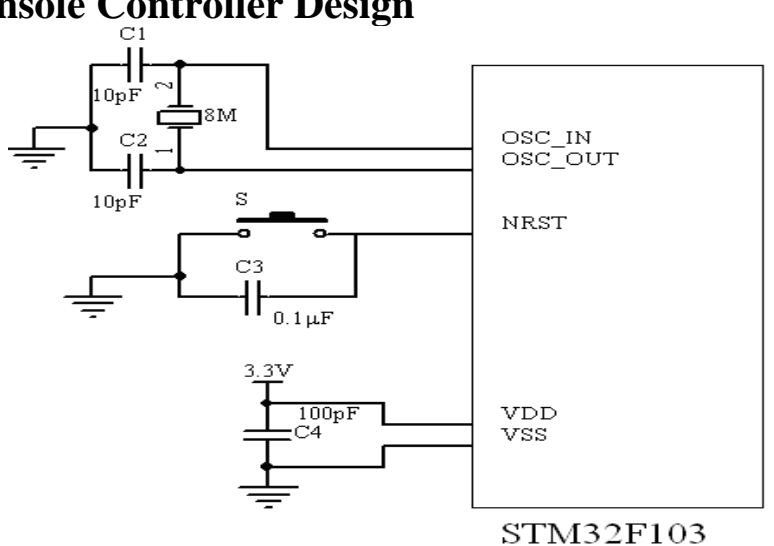

Fig. 2 STM32F103 microcontroller minimum system

Fig. 2 is the schematic diagram of the minimum system circuit of the STM32F103 microcontroller. The minimum system circuit mainly includes three parts: clock, key reset and power supply circuit.

The $8 \mathrm{MHz}$ quartz crystal oscillator and the trimming capacitor are connected between the microcontroller OSC_IN and the OSC_OUT pin to form a stable self-excited oscillator for the STM32 operation.

After the clock circuit works, the NSET pin remains high above $10 \mathrm{~ms}$ and the microcontroller will reset it. In Figure 2, the NSET pin is connected to the $0.1 \mathrm{~F}$ capacitor. The function of this capacitor is to avoid the error reset of some registers in the microcontroller that interfere with the reset end.

In order to corrugated absorption power line, between VCC and access the 100pF decoupling capacitor, the capacitor as energy storage capacitor, and high frequency noise filtering CPU, cut off the channel noise is transmitted through the power supply circuit. The distance between the capacitor and the power source is limited to $20 \mathrm{~mm}$, and better filtering effect can be obtained.

\subsection{Design of Minimum System of Warehouse Controller}

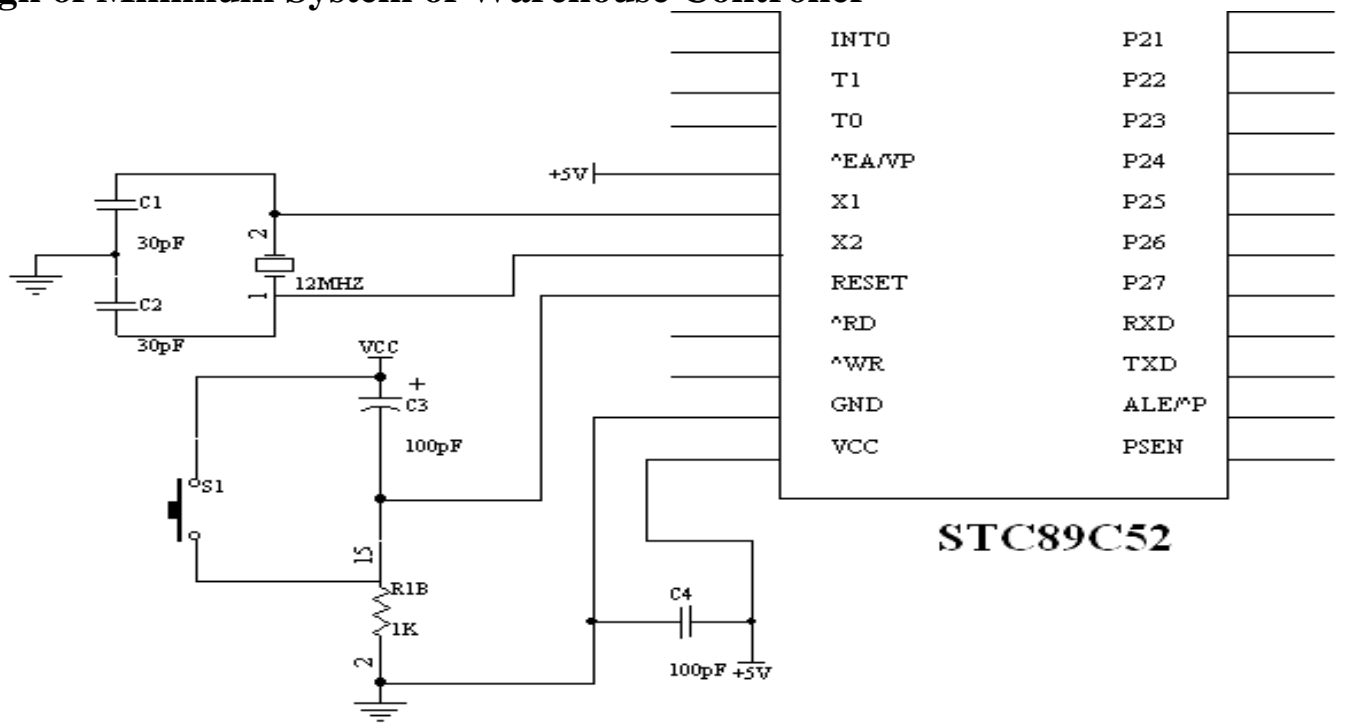

Fig. 3 STC89C52 microcontroller minimum system schematic

As shown in Figure 3, it is the minimum system diagram for the microcontroller. STC89C52 microcontroller system, including the smallest clock circuit and button reset circuit.

The clock circuit is used to generate the clock control signal needed by the microcontroller, and the CPU operates under the control of the clock signal strictly and orderly according to the time sequence command. The STC89C52 has a high gain reverse amplifier (used to form an oscillator). The input of the high gain reverse amplifier is connected to the STC89C52 pin X1 and the output terminal is X2. The quartz crystal oscillator and the trimming capacitor are connected across the two pins, thus forming a stable self-excited oscillator for the STC89C52 operation. 


\subsection{Design of Wireless Transmission Circuit}

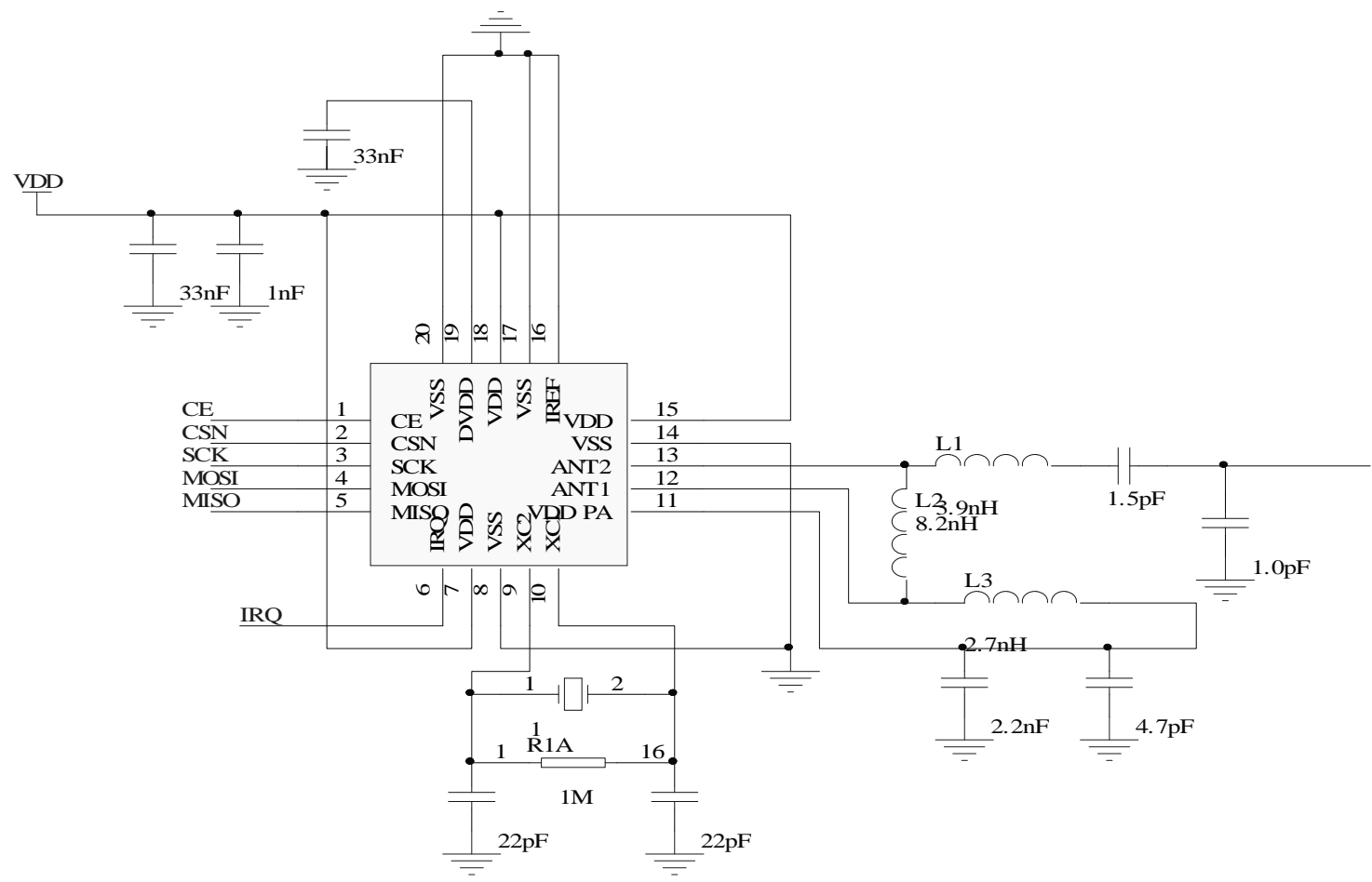

Fig. 4 Schematic diagram of NRF24L01 wireless data transmission circuit

As shown in Figure 4, it is the schematic diagram of the NRF24L01 wireless data transmission circuit. When data is transmitted, the first NRF24L01 is configured to transmit mode: the receiving node address of TX_ADDR and TX_PLD in accordance with the timing data from the SPI port into the NRF24L01 buffer, the TX_PLD must be continuously written in CSN is low, while the TX_ADDR write once in the launch, then CE is set to a high level and at least 10 s. Transmit data 130 s delay after.

When data is received, the NRF24L01 is first configured to receive mode, and then a delay of 130 $s$ is entered into the receiving state to wait for data to arrive. When the receiver detects a valid address and CRC, the packet is stored in the RX FIFO, while the interrupt flag bit RX_DR is set high, the IRQ becomes low, interrupts are generated, and the MCU is notified to fetch the data. If the automatic answer is opened at this time, the receiver enters the transmit state return signal at the same time. If the CE becomes low at last, NRF24L01 enters idle mode 1.

\subsection{The Circuit Design of Console Display}

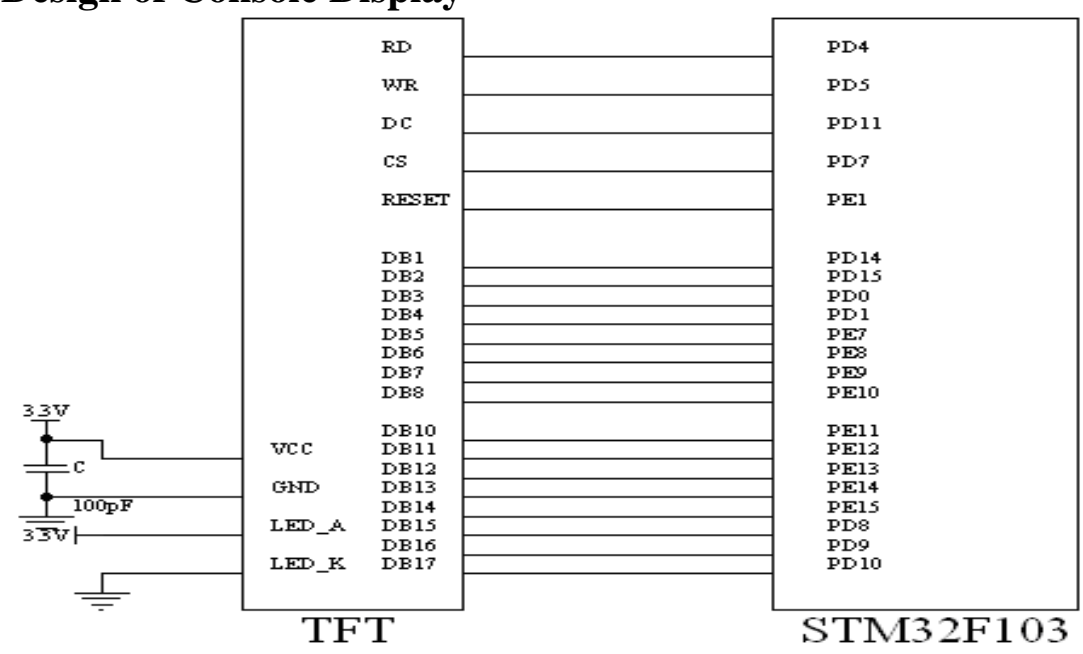

Fig. 5 Color display circuit principle diagram

As shown in Figure 5, it is the schematic diagram of the TFT color touch screen display circuit. The touch screen provides a friendly interface, you can easily enter the warehouse administrator identity 
encoding and password, and open the closed state and enter the time of each warehouse can be displayed in real time, for the administrator to keep abreast of all the warehouse.

\subsection{Warehouse Display Circuit Design}

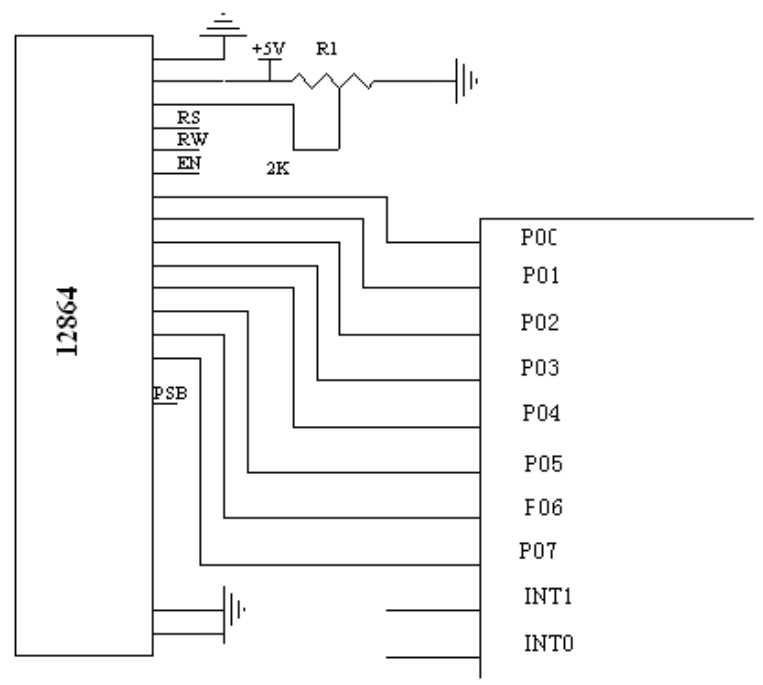

Fig. 6 Schematic diagram of each warehouse display circuit

As shown in Figure 6, the circuit diagram of each warehouse is shown. STC89C52 microcontroller through the P0 port to send information to the LCD12864 data port. In order to realize high-speed data transmission, LCD12864 and SCM adopt parallel communication connection mode.

The warehouse administrator receives the application, through the console CPU generated random password into the warehouse, and the warehouse controller password through the wireless transmission module is sent to the applicant, enter the password, the password will appear in the "*" in the form of a LCD12864 display. When the password is finished, verify, and if correct, open the warehouse door and display the word "correct password" on the LCD12864; otherwise, "password error" is displayed".

\section{Software Design}

\subsection{Console Program Design}

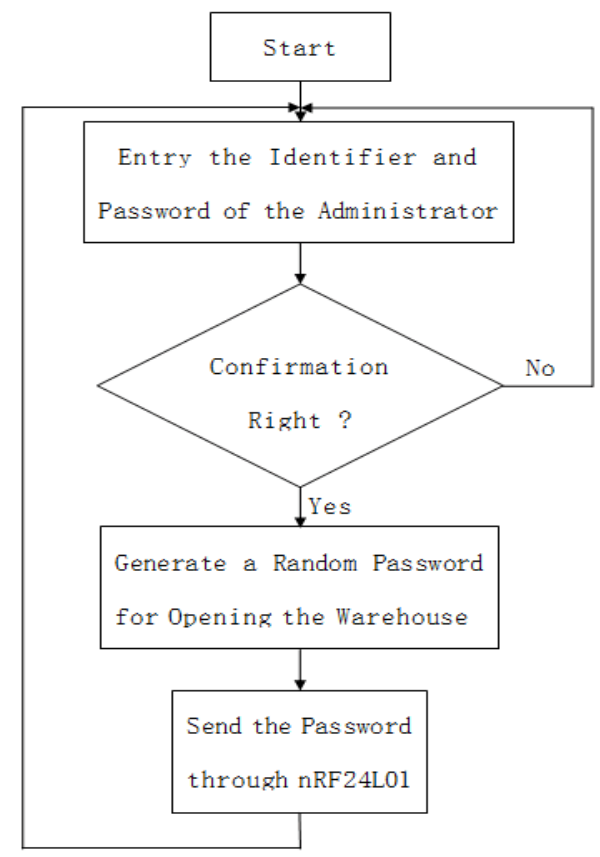

Fig. 7 Console program flow chart 
As shown in Figure 7, the flow chart of the main console program. The working process is as follows: the need to enter the warehouse staff to apply the warehouse administrator, administrator to open the console system, input your identification number and password. After verification, the administrator generates a random password through the operation, and the STM32 microcontroller sends the password through the wireless transmission module NRF24L01 to the STC89C52 microcontroller in the warehouse. After the administrator tells the applicant the password, the applicant needs to enter the random password on the SCM of the warehouse within 3 minutes, and then enter the warehouse after verification. If the applicant does not enter the password within 3 minutes, the password will expire and need to be re applied.

\subsection{Program Design of Warehouse Control Circuit}

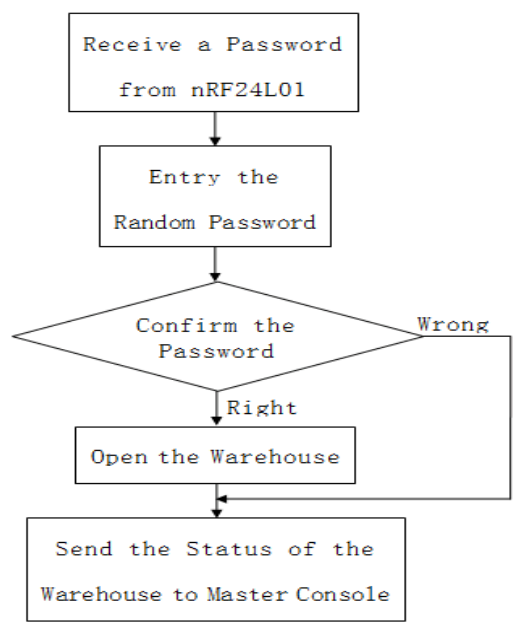

Fig. 8 Flow chart of warehouse control circuit program

The program flow chart of the warehouse control circuit is shown in figure 8. After the warehouse microcontroller receives the random password through the NRF24L01, the applicant may enter the random password that the administrator tells. Through the verification, the warehouse opened, at the same time STC89C52 MCU will warehouse state and enter the time through the wireless module to send back to the main control chip STM32, the administrator to store and view.

\section{Conclusion}

The nRF24L01 wireless transmission module used in this system has a high gain antenna, and the measured distance can be close to 1000 meters. Working in the $2.4 \mathrm{GHz}$ spectrum, signals can penetrate buildings better. With the highest transmission rate can reach $2 \mathrm{Mbps}$, the warehouse administrator only need to enter your ID number and password in the control room on a console, random password to be verified by the applicant told, by its complete open warehouse operation. As long as no special circumstances occur, the administrator will be able to check the status of each warehouse within the main control room, thereby achieving a relaxed and efficient management of each warehouse in the plant area.

\section{Acknowledgments}

This work was financially supported by "2016 the city of Binzhou social science planning and key research project "fund.

\section{References}

[1] Huang Zhiwei, Wang Bing.STM32F, 32 bit ARM microcontroller application design and practice [M]. Beijing: Beihang University press, 2012.

[2] Zhou Xinghua .AVR microcontroller, C language high-level programming design [M]. Beijing: China Electric Power Press, 2011. 
[3] Tan Haoqiang.C programming (Fourth Edition) [M]. Beijing: Tsinghua University press, 2010.

[4] Tong Shibai, Hua Chengying. Fundamentals of Analog Electronic Technology (Fourth Edition) [M]. Beijing: Higher Education Press, 2006.

[5] Yan Shi. Fundamentals of digital electronic technology (Fifth Edition) [M]. Beijing: Higher Education Press, 2006. 